\title{
Cosmic-ray driven dynamo in galactic disks
}

\author{
Michał Hanasz ${ }^{1}$, K. Otmianowska-Mazur ${ }^{2}$, H. Lesch $^{3}$, G. Kowal ${ }^{2,4}$, \\ M. Soida ${ }^{2}$, D. Wóltański ${ }^{1}$ K. Kowalik ${ }^{1}$, R. K. Pawłaszek ${ }^{1}$ \\ and B. Kulesza- $\dot{Z} \mathbf{y d z i k}{ }^{2}$ \\ ${ }^{1}$ Centre for Astronomy, Nicholas Copernicus University, PL-87148 Piwnice/Toruń, Poland, \\ mhanasz@astri.uni.torun.pl \\ ${ }^{2}$ Astronomical Observatory, Jagiellonian University, ul. Orla 171, 30-244 Kraków \\ ${ }^{3}$ Astronomical Observatory, Munich University, Scheinerstr. 1, D-81679, Germany \\ ${ }^{4}$ Department of Physics and Astronomy, McMaster University, 1280 Main St. W., Hamilton,
} ON L8S 4M1, Canada.

\begin{abstract}
We present new developments on the Cosmic-Ray driven, galactic dynamo, modeled by means of direct, resistive CR-MHD simulations, performed with ZEUS and PIERNIK codes. The dynamo action, leading to the amplification of large-scale galactic magnetic fields on galactic rotation timescales, appears as a result of galactic differential rotation, buoyancy of the cosmic ray component and resistive dissipation of small-scale turbulent magnetic fields. Our new results include demonstration of the global-galactic dynamo action driven by Cosmic Rays supplied in supernova remnants. An essential outcome of the new series of global galactic dynamo models is the equipartition of the gas turbulent energy with magnetic field energy and cosmic ray energy, in saturated states of the dynamo on large galactic scales.
\end{abstract}

Keywords. Galaxies: ISM - magnetic fields - ISM: cosmic rays - magnetic fields - MHD

\section{Introduction}

Numerous processes have been proposed to explain initial magnetic fields in early galaxies. However all these processes, like phase transitions in the early universe or Biermann battery in protogalactic objects provide only very week magnetic fields of the order of $B \sim 10^{-20} \mathrm{G}$ at the beginning of galactic evolution. On the other hand, Rees (1987) proposed that initial magnetic fields in galaxies might have been generated in first stars and then scattered in the interstellar medium (ISM) by SN explosions, and subsequently amplified in plerionic (Crab-type) supernova remnants (SNRs). The mean magnetic field on the galactic scale has been estimated to be of the order of $10^{-9} \mathrm{G}$. Radio observations (see the review by $\mathrm{R}$. Beck, this volume) indicate that typical contemporary magnetic fields in spiral galaxies are of the order of few up to few tens of $\mu \mathrm{G}$, which means that magnetic fields have undergone amplification by at least four orders of magnitudes within the galactic lifetime. Therefore, a model for efficient magnetic field amplification during galactic evolution is necessary. The standard model of magnetic field amplification in disk galaxies is based on the theory of turbulent mean field dynamo (see Widrow 2002 for a recent review).

The mean-field dynamo theory has been successful in explaining magnetic fields in various astrophysical objects, however, the classical, kinematic dynamo seems to be rather slow in galaxies. Magnetic field amplification timescale $t_{\text {dynamo }} \sim(0.5 \div 1) \times 10^{9}$ yrs is too long to explain $\sim 1 \mu \mathrm{G}$ magnetic fields in galaxies which are only few $10^{9}$ yrs old 
$(z \sim 1 \div 3)$ (Wolfe, Lanzetta \& Oren 1992, Kronberg et al. 2008). These circumstances seem to indicate a need of an alternative approach. We suggest that direct numerical MHD modeling of ISM dynamics, through considerations of detailed physical processes, involving major ISM components: gas, magnetic fields and cosmic rays, is necessary to follow magnetic history of galaxies.

\section{Cosmic Rays in the interstellar medium}

The dynamical role of CRs was recognized for the first time by Parker (1966), who noticed that a vertically stratified ISM, consisting of thermal gas magnetic field and CRs is unstable due to buoyancy of the weightless components: magnetic fields and CRs. The CR component appears to be an important ingredient of this process. According to the diffusive shock acceleration models CRs are continuously supplied to ISM by SN remnants. Therefore, strong buoyancy effects due to CRs are unavoidable.

Theories of diffusive shock acceleration predict that about $10 \%$ of the $\sim 10^{51} \mathrm{erg}$ of the SN II explosion energy is converted to the CR energy (see e.g. Jones et al 1998 and references therein). Observational data indicate that gas, magnetic fields and CRs appear in approximate energetic equipartition, which means that all the three components are dynamically coupled. Moreover, numerical experiments by Giaccalone \& Jokipii (1999) indicate that CRs diffuse anisotropically in the ISM, along the mean magnetic field direction.

\section{Cosmic Ray transport}

To incorporate CR propagation in MHD considerations we use the diffusion - advection equation (e.g. Schlickeiser \& Lerche 1985)

$$
\begin{array}{r}
\frac{\partial e_{\mathrm{cr}}}{\partial t}+\boldsymbol{\nabla}\left(e_{\mathrm{cr}} \boldsymbol{v}\right)=-p_{\mathrm{cr}} \boldsymbol{\nabla} \cdot \boldsymbol{v}+\boldsymbol{\nabla}\left(\hat{K} \boldsymbol{\nabla} e_{\mathrm{cr}}\right) \\
+\mathrm{CR} \text { sources (SN remnants) }
\end{array}
$$

where the diffusion term in written in the tensorial form to account for anisotropic diffusivity of CRs. The sources of CRs on the rhs. of Eqn. 3.1 correspond to the CR production in Supernova remnants.

The dynamics of ISM including the energetics of CRs can be now described by the CR transport Eqn. (3.1) and the system of MHD equations. We note that in the presence of cosmic rays additional source term: $-\nabla P_{\mathrm{CR}}$ should be included in the gas equation of motion (see e.g. Berezinski et al. 1990), in order to incorporate the effects of CRs on gas dynamics. The cosmic ray diffusion-advection Eqn. (3.1) has been supplemented to the MHD algorithm of Zeus-3D MHD code by Hanasz \& Lesch (2003a) with the aim of studying ISM physical processes, e.g. Parker instability, in which CRs play a dynamically important role.

\section{CR-driven dynamo}

Referring back to the issue of galactic magnetic fields, we are primarily interested in processes leading to the amplification of large scale magnetic fields. The original idea of CR-driven dynamo has been raised by Parker (1992). Our first local (shearing-box) CRdriven dynamo numerical experiments (Hanasz et al. 2004, 2006; Otmianowska-Mazur et al. 2007) rely on the following ingredients: (1.) the cosmic ray component described by the diffusion-advection transport equation (3.1), including localized sources of cosmic 

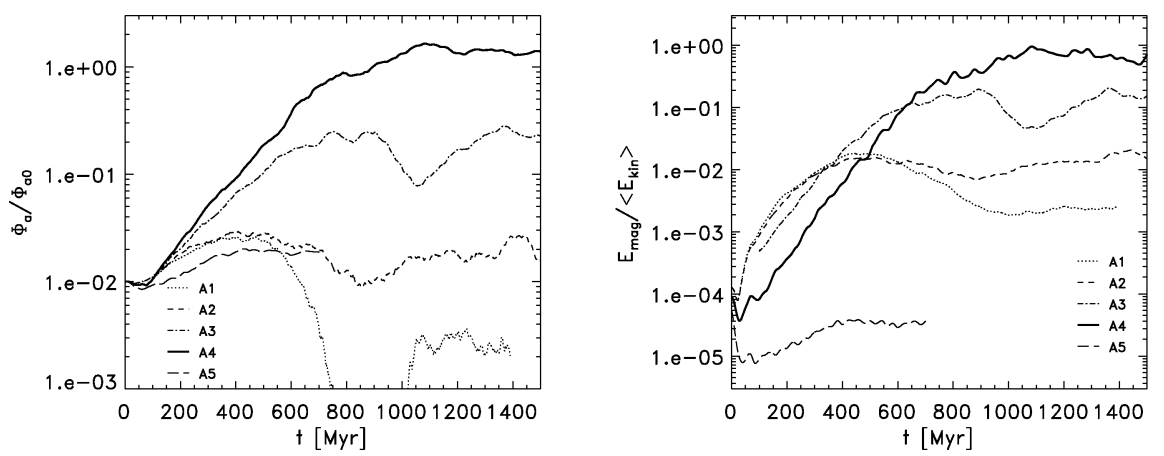

Figure 1. Time evolution of azimuthal magnetic flux and total magnetic energy for different values of magnetic diffusivity in simulation series $\mathrm{A}$. The curves represent respectively cases of $\eta=0$ (A1), $\eta=1$ (A2), $\eta=10$ (A3), $\eta=100$ (A4) and $\eta=1000$ (A5) in units $\mathrm{pc}^{2} \mathrm{Myr}^{-1}$.

rays - supernovae remnants, exploding randomly in the disk volume, while SN shocks and thermal effects are neglected (see Gressel et al. 2008a, 2008b) for a recent dynamo model relying on the thermal energy output from SNe); (2.) resistivity of the ISM (see Hanasz, Otmianowska-Mazur and Lesch 2002), leading to topological evolution of magnetic fields; (3) shearing boundary conditions (Hawley, Gammie and Balbus 1995) together with Coriolis and tidal forces, aimed at modeling of differentially rotating disks in the local approximation; (4) realistic vertical disk gravity and rotation following the model of ISM in the Milky Way by Ferrière (1998).

In the most recent paper (Hanasz et al. 2009) we present a parameter study of the $\mathrm{CR}$-driven dynamo model by examining the dependence of magnetic field amplification on magnetic diffusivity, supernovae rate determining the $\mathrm{CR}$ injection rate, temporal modulation of SN activity, grid resolution, and CR diffusion coefficients. We find the dominating influence of magnetic diffusivity (treated as a free parameter), among other parameters, (Fig. 1) on the efficiency of magnetic field amplification.

The fastest magnetic field amplification, observed in the experiments, coincides with the magnetic diffusivity comparable to $\eta \simeq \frac{1}{3} \eta_{\text {turb }}($ obs $)$, where $\eta_{\text {turb }}($ obs $) \simeq \frac{1}{3} 100 \mathrm{pc} \times$ $10 \mathrm{~km} \mathrm{~s}^{-1} \simeq 10^{26} \mathrm{~cm} \mathrm{~s}^{-1}$. The question of which physical process can be responsible for that large magnetic diffusivity coefficients points out towards the current studies on magnetic reconnection (Lazarian et al. 2004, Cassak et al. 2006).

To investigate observational properties of current dynamo models we perform a series of numerical simulations, placing the local shearing-boxes at different galactocentric radii. We replicate the contents of local cubes into rings, and then combine rings to build up a disk (Otmianowska-Mazur et al. 2009). A synthetic polarization radio-map of synchrotron emission constructed, on the base of simulated magnetic fields and CR distribution is shown in Fig. 4.

A polarized emission map together with superposed polarization vectors of synchrotron emission of an edge-on galaxy NGC 5775 is shown for comparison. As it is apparent the $X$-shaped structures of magnetic field, present in the real galaxy, form also in the synthetic radio image. The presented results demonstrate that the CR-MHD simulations provide a way of verification of $\mathrm{CR}$-dynamo models against the observational data.

To summarize the overall outcome of the local simulations of CR-driven dynamo, we note that the model provides efficient amplification of large-scale magnetic fields, which 

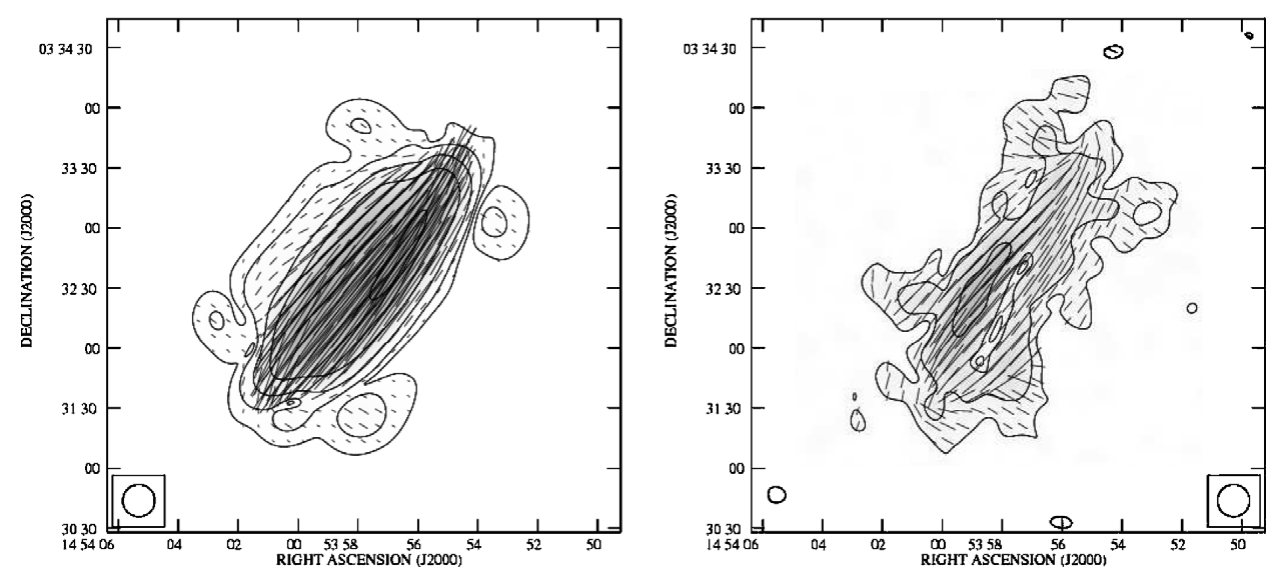

Figure 2. Synthetic radio-map of polarized radio emission for our model at the inclination $i=80^{\circ}$ and a map of real galaxy NGC 5775 .

attain, in the saturated state, equipartition with turbulent gas motions. However,we note also a drawback of the local models, since the CR energy density remains more than an order of magnitude larger than gas and magnetic energy densities. We recognize that improvements of the current models are necessary, since the possible reason of the excess of cosmic rays may result from the effect of trapping of cosmic rays by predominantly horizontal magnetic field in the computational box which is periodic in horizontal directions. Therefore, global galactic disk simulations should be performed to enable escape of CRs along the predominantly horizontal magnetic field in the galactic plane, in order to avoid the CR excess.

\section{Global disk simulations}

Recently we started a new series of global simulations of CR-driven dynamo with the aid of PIERNIK MHD code (see Hanasz et al. 2009a, 2009b, 2009c, 2009d), which is a grid-based MPI parallelized, resistive MHD code based on the Relaxing TVD (RTVD) scheme by Jin \& Xin (1995) and Pen et al. (2003). The original scheme is extended to deal with the diffusive CR component (see Hanasz \& Lesch 2003a).

In Fig. 3 we show results of one of the first semi-global simulations of CR-driven dynamo. The simulations have been performed for a quarter of galactic disk resembling Milky Way, with parallel CR diffusion coefficient $K_{\|}=3 \times 10^{28} \mathrm{~cm}^{2} \mathrm{~s}^{-1}$, in a computational domain $20 \mathrm{kpc} \times 20 \mathrm{kpc} \times 20 \mathrm{kpc}$, and resolution of $400 \times 400 \times 160$ grid cells, in 16 MPI blocks.

The semi-global simulations confirm the fast amplification rate of the mean magnetic field: growth time of the mean magnetic flux is approximately $210 \mathrm{Myr}$ in the whole galaxy, which is close to the galactic rotation period near the orbit of Sun. As expected, the global simulations introduce a significant improvement with respect to the former shearing-box simulations. It is apparent in Fig. 3 that the ratio of magnetic to cosmic ray energy is close to one in the synchrotron emitting volume (compare panels 4.and 5.), while over-equipartition of CR energy with respect to magnetic energy still holds outside the disk.

The latest development of our CR-dynamo model is a fully global galactic disk simulation (see the complementary paper by Hanasz, et al. this volume), where we demonstrate that dipolar magnetic fields supplied on small SN-remnant scales, can be amplified 


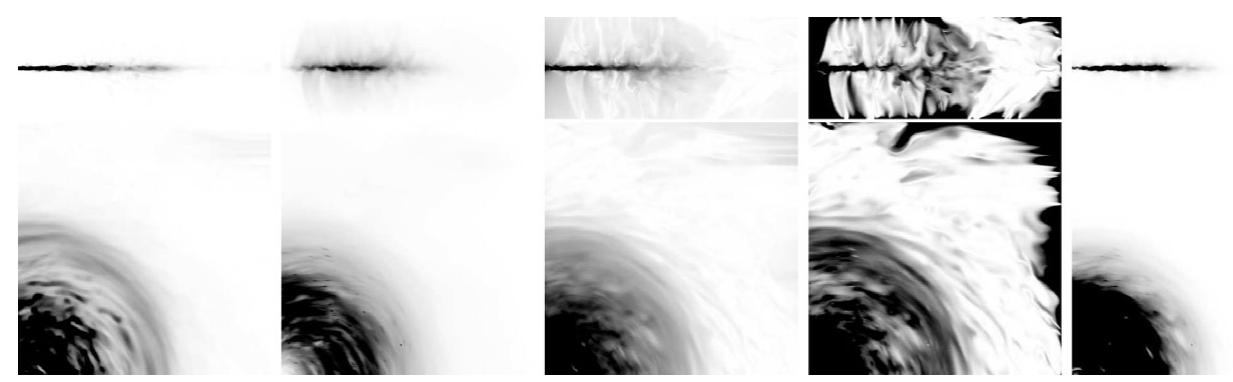

Figure 3. Horizontal and vertical slices through the computational box for a simulation of CR-driven dynamo in a semi-global galactic disk. The color maps reflect: 1: gas density, 2: CR energy density, 3: magnetic field strength, 4 . the ratio of magnetic to CR energy densities and 5 . synchrotron emissivity for a saturated state of the dynamo. The gray-scales in all panels, except 4. are chosen arbitrarily. The darker shades correspond to larger values of displayed quantities. The greyscale of panel 4 . is such that black areas represent values of $e_{\mathrm{mag}} / e_{\mathrm{cr}} \simeq 1$.

exponentially by the $\mathrm{CR}$-driven dynamo to the present equipartition values, and transformed simultaneously to large galactic-scales.

\section{Conclusions}

We have shown that the CR contribution to the dynamics of ISM, studied by means of CR-MHD simulations, in both local and global scales, leads to a very efficient magnetic field amplification in galactic disks.

The cosmic ray driven dynamo amplifies efficiently galactic magnetic fields on a timescale of galactic rotation. The resulting growth of the large-scale magnetic field by 4 orders of magnitude within 2 Gyr (Hanasz et al. 2004), is fast enough to expect $\sim 1 \mu \mathrm{G}$ magnetic field in galaxies at $z \sim 1 \div 3$.

We point out the advantage of global disk models, which on the contrary to shearingbox models, provide CR energy equipartition with magnetic field in the synchrotron emitting part of the disks. This work was supported by Polish Ministry of Science and Higher Education through the grants 92/N-ASTROSIM/2008/0 and PB 0656/P03D/2004/26 and by Nicolaus Copernicus University through Rector's grant No. 516-A.

\section{References}

Beck R. 2008, ApछSSS, in press (arXiv:astro-ph/0711.4700)

Berezinskii, V. S., Bulanov, S. V., Dogiel, V. A., Ginzburg, V. L., \& Ptuskin, V. S. Astrophysics of cosmic rays, Amsterdam: North-Holland, 1990.

Cassak, P. A., Drake, J. F., \& Shay, M. A. 2006, ApJ 644, L145

Giacalone, J. \& Jokipii, R. J. 1999, ApJ 520, 204

Ferrière, K. 1998, ApJ 497, 759

Gressel, O., Ziegler, U., Elstner, D., \& Rüdiger, G. 2008, AN 329, 61

Gressel, O., Elstner, D., Ziegler, U., \& Rüdiger, G. 2008, $A \& A$ A 486, L35

Hanasz, M., Otmianowska-Mazur, K., \& Lesch, H. 2002, A\&\&A 386, 347

Hanasz, M. \& Lesch, H. 2003, A\&SA 412, 331

Hanasz, M., Kowal, G., Otmianowska-Mazur, K., \& Lesch, H. 2004, ApJ 605, L33

Hanasz, M., Kowal, G., Otmianowska-Mazur, K., \& Lesch, H. 2006, AN 327, 469

Hanasz, M., Otmianowska-Mazur, K., Kowal, G., \& Lesch, H. 2009, A\&A, in press (arXiv:astro$\mathrm{ph} / 0812.3906)$ 
Hanasz, M., Kowalik, K., Wóltański, D., \& Pawłaszek, R. K. 2009, in: K. Goździewski et al. (eds.) Extrasolar planets in multi-body systems: theory and observations, EAS Publications Series (submitted, arXiv:astro-ph/0812.2161)

Hanasz, M., Kowalik, K., Wóltański, D., \& Pawłaszek, R. K. 2009, in: K. Goździewski et al. (eds.) Extrasolar planets in multi-body systems: theory and observations, EAS Publications Series (submitted, arXiv:astro-ph/0812.2799)

Hanasz, M., Kowalik, K., Wóltański, D., \& Pawłaszek, R. K., 2009, in: M. de Avillez et al. (eds.), The Role of Disk-Halo Interaction in Galaxy Evolution: Outflow vs Infall?, (submitted, arXiv:astro-ph)

Hanasz, M., Kowalik, K., Wóltański, D., \& Pawłaszek, R. K., 2009, in: M. de Avillez et al. (eds.), The Role of Disk-Halo Interaction in Galaxy Evolution: Outflow vs Infall?, (submitted, arXiv:astro-ph)

Hawley, J. F., Gammie, C. F., \& Balbus, S. A. 1995, ApJ 440, 442

Jones, T. W., Rudnick, L., Jun, B.-I., Borkowski, K. J., Dubner, G., Frail, D. A., Kang, H., Kassim, N. E., \& McCray, R. 1998, PASP 110, 125

Jin, S. \& Xin, Z. 1995, Comm. Pure Appl. Math. 48, 235

Kronberg, P. P., Bernet, M. L., Miniati, F., Lilly, S. J., Short, M. B., \& Higdon, D. M. 2008, ApJ 676, 70

Lazarian, A., Vishniac, E. T., \& Cho, J. 2004, ApJ 603, 180L

Otmianowska-Mazur, K., Kowal, G., \& Hanasz, M., 2007, ApJ 668, 1100

Otmianowska-Mazur, K., Soida, M., Kulesza-Żydzik, B., Hanasz, M., \& Kowal, G. 2009, ApJ, in press (arXiv:astro-ph/0812.2150)

Parker, E. N. 1966, ApJ 145, 811

Parker, E. N. 1992, ApJ 401, 137

Pen, U.-L., Arras, P., \& Wong, S. 2003, ApJS 149, 447

Rees, M. J. 1987, QJRAS 28, 197

Ryu, D., Kim, J., Hong, S. S., \& Jones, T. W. 2003, ApJ 589, 338

Schlickeiser, R. \& Lerche, I. 1985, A\& A 151, 151

Widrow, L. M. 2002, Rev. Mod. Phys. 74, 775

Wolfe, A. M., Lanzetta, K. M., \& Oren, A. L. 1992, ApJ 388, 17

\section{Discussion}

GRESSEL: 1) In your simulations, what is the ratio of the turbulent and regular magnetic field strength? 2) With a strong turbulent component of the field, wouldn't you expect the cosmic-ray diffusion to be suppressed/diminished due to the highly tangled field geometry?

HANASz: 1) The ratio of the turbulent to regular magnetic field strength is of the order of 1, i.e. unity. 2) The buoyancy of cosmic rays continuously supplied to the system, and trapped by the tangled magnetic field, becomes strong enough to stretch magnetic field lines in the vertical direction. Then, the magnetic field should become less tangled, allowing cosmic rays to leave the disk along the stretched-out vertical field lines. 